\title{
Beyond Programming: A Computer-Based Assessment of Computational Thinking Competency
}

\author{
RINA P. Y. LAI, Faculty of Education, University of Cambridge, Cambridge, United Kingdom
}

\begin{abstract}
Computational Thinking (CT), entailing both domain-general and domain-specific skills, is a competency fundamental to computing education and beyond. However, as a cross-domain competency, appropriate assessment design and method remain equivocal. Indeed, the majority of the existing assessments have a predominant focus on measuring programming proficiency and neglecting other contexts in which CT can also be manifested. To broaden the promotion and practice of $\mathrm{CT}$, it is necessary to integrate diverse problem types and item formats using a competency-based assessment method to measure CT. Taking a psychometric approach, this article evaluates a novel computer-based assessment of CT competency, Computational Thinking Challenge. The assessment was administered to 119 British upper secondary school students $(M=$ $16.11 ; S D=1.19)$ with a range of prior programming experiences. Results from several reliability analyses, a convergent validity analysis, and a Rasch analysis, provided evidence to support the quality of the assessment. Taken together, the study demonstrated the feasibility to expand from traditional assessment methods to integrating multiple contexts, problem types, and item formats in measuring CT competency in a comprehensive manner.
\end{abstract}

\section{CCS Concepts: • Social and professional topics $\rightarrow$ Computational thinking;}

Additional Key Words and Phrases: Computational thinking competency, computer-based assessment, computing education, problem-solving

\section{ACM Reference format:}

Rina P. Y. Lai. 2021. Beyond Programming: A Computer-Based Assessment of Computational Thinking Competency. ACM Trans. Comput. Educ. 22, 2, Article 14 (November 2021), 27 pages.

https://doi.org/10.1145/3486598

\section{INTRODUCTION}

In the last decade, computing activities have become ubiquitous in the classroom, either being embedded within a subject domain or integrated as cross-subject activities. One of the central goals is to promote computational thinking (CT) such that learners are equipped with fundamental skills to solve complex real-world problems. With this objective, there is an increasing demand to better understand how, and to what extent, learners have developed CT. Due to the paucity of reliable and valid CT assessments in the literature, this need has become a challenge to many educators and researchers [1]. Moreover, despite the efforts being put into the development of $\mathrm{CT}$ assessments, there remains ambiguity regarding appropriate methods and approaches. Indeed,

Author's address: R. P. Y. Lai, Faculty of Education, University of Cambridge, 184 Hills Rd, Cambridge CB2 8PQ, United Kingdom; email:pyrl3@cam.ac.uk.

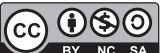

(C) 2021 Copyright held by the owner/author(s).

1946-6226/2021/11-ART14

https://doi.org/10.1145/3486598
}

This work is licensed under a Creative Commons Attribution-Share Alike International 4.0 License. 
since the inception of the term, diverse perspectives have been proposed, leading to the development of different types of assessment tools, with the majority targeting programming proficiency [3]. Although measuring CT in block-based programming environments is a common approach, other areas in which CT can be demonstrated have been neglected, such as problem-solving and cross-domain contexts. Hence, while a programming-centric approach is sufficient in measuring $\mathrm{CT}$ in a single specific context, it narrows our understanding of CT, particularly with the emerging view that $\mathrm{CT}$ is a competency [3]. This is the case as the nature of competency demands the use of a diverse range of computing-relevant tasks-including but not limited to programming. This article is motivated to expand the current scope of CT assessment methods. In so doing, two broader families in CT conceptualization are reviewed, current assessments are appraised and finally, a novel computer-based assessment of CT competency is evaluated from a psychometric approach.

\section{RELATED WORK}

\section{Computational Thinking: From Programming to Cross-Domain Problem-Solving}

There is a long-standing belief that interacting with a computer can impact the way learners learn, enhance thinking skills, and alter the way knowledge is accessed [4]. Through this interaction, learners are prompted to think computationally and innovatively to construct meaningful products [5]. Emerging from this perspective is an early view of CT, one that situates the construct within learning contexts that surround computing, programming, and the use of technology. For example, based on their work in Scratch, Brennan \& Resnick [6] highlight three dimensions in their $\mathrm{CT}$ framework in relation to programming: computational concepts, practices, and perspectives. Similarly, Voskoglou \& Buckley [7] consider CT as a type of analytical thinking that associates with programming and coding, useful for complex real-world problems. Like these authors, Grover \& Pea [8] contend that programming is an important tool to facilitate cognitive tasks in CT and a means to demonstrate CT competencies. However, Sysło \& Kwiatkowska [9] and Csizmadia et al. [10] caution that the conceptualization of CT should not be limited to programming alone but rather be considered within computing principles and beyond. Voogt et al. [11] explain that the view of $\mathrm{CT}$ being equated to programming stems from the extensive studies that consider it within the computer science literature.

In the last decade there is an emerging view brought forth by Wing's [12] seminal article, "Computational Thinking", that CT can and should be considered in a more general manner with the focus on problem-solving [13-15]. That is, CT as a general set of cognitive processes can be used by everyone across contexts- with or without machines [12]. Just as basic skills like reading and arithmetic can be used in diverse situations, CT is considered a cognitive tool useful in many areas and is imperative for effective problem-solving $[12,14]$. There are two premises under this perspective: (1) CT benefits everyone in the technological world and is, therefore, an important skill to be developed for all learners, and (2) it facilitates advanced problem-solving strategies across disciplines [16]. In this regard, CT may be considered a subset of domain-general problem solving skills that is relevant to computing [19]. Not only is CT theoretically important to problem-solving, but recent studies have also provided some empirical support. For example, Román-González and colleagues [17] have shown that amongst various cognitive factors, CT has the strongest correlation with problem-solving ability. Moreover, in a cluster analysis of a teacher survey, Lai [19] has also shown that problem-solving and cross-disciplinary skills were two main clusters that explained how computer science educators conceptualized CT. On a more practical note, fostering problem-solving skills has been identified as a shared goal and rationale for integrating CT in the curriculum in the 13 countries reviewed in Bocconi et al. [18]. As such, the conceptualization of CT has observed an evolution in the literature: from that of programming proficiency to cross-domain 
problem-solving skills. Hence, CT competency encompasses a broad range of and integration between domain-general problem-solving skills and domain-specific computing principles useful in diverse contexts and can be expressed in dynamic ways [19].

Despite the different contexts in which researchers conceptualize CT, the perspective as to the cognitive skills CT entails is largely shared. For example, in summarizing previous work of computer science researchers and educators, abstractions/ pattern generalization, algorithmic thinking, problem decomposition, and debugging (along with other CT programming concepts) are considered to be key CT components [8]. Likewise, Selby \& Woollard [20] conducted a literature analysis and extracted the same set of skills. The conceptualizations of these authors align with Wing's [12] suggestion: working and thinking between multiple levels of abstraction; identifying algorithmic approaches to problems; utilizing problem- decomposition in large and complex problems. Barr \& Stephenson [21] also identify the same group of skills but extend their perspectives beyond computer science education to math, science, socials studies, and language arts. That is, they consider CT skills, important to computer science education, to be also relevant across subjects. In their report, Boccino et al. [18] summarize cognitive skills that are shared amongst different researchers; they have also identified the aforementioned set of skills to be key. Given the extensive discussion of what CT comprises, there appears to be a consensus that it entails at least five key cognitive processes. They are summarized and described in Table 1.

\section{Computational Thinking Assessment}

2.2.1 Domain-Specific Assessment of CT. Existing CT assessments have encapsulated aspects of the aforementioned nature of CT. However, as demonstrated by a recent systematic review, the majority of the assessments remain domain-specific, with a focus on computer science and programming [25]. For example, in their systematic mapping, Alves \& Hauck [26] note the lack of diversity in assessment approaches as most tools focus on blocked-based visual programming languages. Wiebe et al. [25] consider these assessments as "code-centric" assessments.

A common and widely used tool is Dr. Scratch, a web-application that automatically assesses CT through code written in Scratch (https://scratch.mit.edu/), a child-friendly graphical programming language [27]. It evaluates student projects, provides feedback, and assigns scores based on the CT skills demonstrated in the projects. The score is based on seven CT components: abstraction/ problem decomposition, logical thinking, synchronization, parallelism, algorithmic thinking, as well as interactivity and data representation. A low CT score indicates that a learner is a "novice programmer" and a high score indicates expertise in programming [27]. Hence, it is assumed that $\mathrm{CT}$ proficiency and coding proficiency are equivalent to the extent that $\mathrm{CT}$ is assessed through coding projects completed in Scratch. Nonetheless, as Scratch is a common tool in the classroom, Dr. Scratch can be easily integrated into the lessons to evaluate learning progression.

Similarly, the Fairy Performance Assessment was designed specifically for two programming environments, Storytelling Alice and Alice 2.2 [29]. Learners use the drag-and-drop graphical programming language to code the behaviors of characters in the environment to illustrate a story. In particular, algorithmic thinking, abstraction, and modeling are measured by three independent assessment tasks that are embedded in a narrative framework of a story. The assessment was found to be suitable for learners with a range of CT skills in middle school.

An assessment that is closely embedded in computer science education is the Real-Time Evaluation and Assessment of Computational Thinking system. It was designed to help computer science teachers to evaluate which CT concepts learners have mastered or have not mastered as they code in real-time [30]. The collected real- time data are recorded into the database to be analyzed through three stages, including the extraction and interpretation of the semantic meaning of the 
Table 1. Cognitive Components of CT

\begin{tabular}{|c|c|c|}
\hline Cognitive components & Description & Demonstrated skills \\
\hline \multirow[t]{3}{*}{ Abstraction } & $\begin{array}{l}\text { The process of creating something simple from } \\
\text { something complicated by filtering out irrelevant details; } \\
\text { finding relevant patterns and separating ideas from } \\
\text { tangible details [22]. }\end{array}$ & $\begin{array}{l}\text { - Identify key characteristics of } \\
\text { a problem } \\
\text { - Distinguish relevant and } \\
\text { irrelevant information }\end{array}$ \\
\hline & $\begin{array}{l}\text { Reducing details so as to target relevant information for } \\
\text { problem-solving [8]. }\end{array}$ & $\begin{array}{l}\text { - Filter out irrelevant } \\
\text { information }\end{array}$ \\
\hline & $\begin{array}{l}\text { Wing [12] and Grover \& Pea [8] argue that the essence } \\
\text { of CT is abstraction. }\end{array}$ & \\
\hline \multirow[t]{2}{*}{ Algorithmic Thinking } & $\begin{array}{l}\text { The practice of writing step-by-step specific and explicit } \\
\text { instructions for carrying out a task or solving a problem } \\
\text { [22]. }\end{array}$ & $\begin{array}{l}\text { - Explicitly state the } \\
\text { algorithmic steps }\end{array}$ \\
\hline & $\begin{array}{l}\text { Kazimoglu et al [23] argue that the selection of } \\
\text { appropriate algorithmic techniques is a crucial part of } \\
\text { CT. Grover \& Pea [8] argue algorithms are tools to } \\
\text { express computational solutions. }\end{array}$ & $\begin{array}{l}\text { - Identify the most efficient } \\
\text { algorithms for a given } \\
\text { problem }\end{array}$ \\
\hline \multirow[t]{2}{*}{ Problem Decomposition } & $\begin{array}{l}\text { The process of breaking down problems into smaller } \\
\text { parts that may be easier to solve [22]. }\end{array}$ & $\begin{array}{l}\text { - Break down a bigger problem } \\
\text { into smaller and simpler parts }\end{array}$ \\
\hline & $\begin{array}{l}\text { Wing [12] suggests that CT is using problem } \\
\text { decomposition when solving or designing a large } \\
\text { complex problem. }\end{array}$ & \\
\hline \multirow[t]{3}{*}{ Generalization } & $\begin{array}{l}\text { To progress from details and parts of a specific solution } \\
\text { to broader applications and problems }[20]\end{array}$ & $\begin{array}{l}\text { - Apply useable parts of a } \\
\text { previous solution to a similar } \\
\text { or new problem }\end{array}$ \\
\hline & $\begin{array}{l}\text { Finding similarities and connections between problems } \\
\text { so as to solving a new problem efficiently [10]. }\end{array}$ & $\begin{array}{l}\text { - Identify commonalities and } \\
\text { patterns between problems }\end{array}$ \\
\hline & $\begin{array}{l}\text { Selby \& Woollard [20] recognize generalization to be a } \\
\text { fundamental component of } \mathrm{CT} \text { in their literature analysis } \\
\text { based on its frequency and consistency in the literature. }\end{array}$ & \\
\hline \multirow[t]{3}{*}{ Debugging } & $\begin{array}{l}\text { Identifying and correcting errors to arrive at a correct } \\
\text { solution [24] }\end{array}$ & $\begin{array}{l}\text { - Evaluate whether a solution } \\
\text { is good or not }\end{array}$ \\
\hline & $\begin{array}{l}\text { Analyzing and evaluating using testing, tracing, and } \\
\text { logic to predict and verify results [10] }\end{array}$ & - Identify errors and mistakes \\
\hline & Wing [12] identifies debugging as a key element of CT. & $\begin{array}{l}\text { - Make corrections when } \\
\text { mistakes are made }\end{array}$ \\
\hline
\end{tabular}

codes. As it measures $\mathrm{CT}$ in real time while lessons are in progress, it has the potential for teachers to inform and intervene when errors are made, thus improving the quality of teaching and learning.

Likewise, focusing on introductory programming modules, the FACT (Foundations for Advancing Computational Thinking) assessment was designed to utilize "systems of assessments" to capture learners' CT programming skills [31]. These systems include open-ended programming tasks, Parson's puzzles, multiple-choice items, block- and text-based programming tests, as well as student projects and surveys. This multifaceted approach allows the evaluation of both the cognitive and non-cognitive aspects of $\mathrm{CT}$, with a large focus on programming.

While the above assessments target a specific representational form (e.g., blocked-based or textbased programming language), the commutative assessment uses multiple-choice items and includes both forms [32]. This allows researchers to evaluate the difference in performance patterns and misconception frequency between the different forms of items. Hence, it is particularly beneficial for pre-post-test study design and for the purpose of understanding proficiency, transfer effect, and progression between the two forms. 
2.2.2 Domain-General Assessment of CT. The predominant focus on programming in the existing assessment tools has motivated several researchers to suggest extending CT assessments to broader contexts, beyond programming $[13,25]$. Although scarce, several assessments were designed to measure CT in a context-neutral manner. For example, the Computational Thinking Test (CTt), designed for middle-school students, measures CT in a somewhat decontextualized manner using Scratch-like blocks. Its items focus on concepts of computing and logic-syntax of programming languages rather than cognitive components, such as basic sequences, loops, iteration, conditionals, functions, and variables [33]. Designed as a scale, it is a psychometrically sound measurement tool as it is one of the few CT assessments that has conducted several stages of validity analysis [34] and in different countries [35]. However, despite its non-reliance on a specific programming language or environment, its items are largely focused on programming concepts.

Recently, an assessment that focuses on computational thinking practices in primary school students was developed and evaluated [36]. The assessment measures four dimensions of CT practices adopted from [6], namely testing/ debugging, algorithmic thinking, reusing/ remixing, and abstracting/ modularizing. The items are situated within general problem-solving contexts and are not specific to any programming languages. Therefore, it could be used with other programmingcentric assessments in measuring CT in a comprehensive manner or used outside the context of computing education. In particular, the authors suggest its feasibility in measuring CT in largescale studies, beyond the use of qualitative or artifact-based assessments.

Another tool that is independent of any programming languages, environments, or curriculum is the Bebras ${ }^{\circledR}$ Computing Challenge (Bebras.org), an international informatics contest. Although designed as contest, Bebras ${ }^{\circledR}$ has been used to assess computing or CT approaches to problemsolving [37, 38]. Due to its context-neutral nature, it has been used to detect CT transfer skills [39] and CT competencies [40]. Its items are interactive multiple- choice questions; each item measures at least some aspects of CT practices, such as abstraction and algorithmic thinking [41]. Similar to the CTt, Bebras ${ }^{\circledR}$ is also one of the few tools that has gone through initial psychometric analyses [40]. However, recent findings suggest Bebras ${ }^{\circledR}$ cannot detect CT components robustly under a five- factor theoretical model, suggesting these components could be indistinguishable in the tasks [42]. Nonetheless, the contest may help promote engagement in CT and computer science education.

While the above assessments emphasize domain-general CT skills, the CTt has a larger focus on programming in a decontextualized environment whereas the Bebras ${ }^{\circledR}$ targets problem-solving. Recently, Wiebe et al. [27] explored the possibility of combining items from these two assessments by investigating the psychometric properties of a "lean" set of tasks for middle school students. The outcome of the study is a more condensed assessment tool with quality items, reflecting the complementary nature of the tools. This assessment method is particularly useful for researchers who wish to assess CT in a more holistic manner.

Other than the above assessments, Rowe et al. [43] developed a game-based learning assessment of implicit CT skills in the puzzle game, Zoombinis. The idea of implicit CT skills surrounds everyday activities that are not usually expressed explicitly by learners in a formal setting, such as formal testing or in schoolwork [43]. Hence, knowledge in CT is inferred by learners' gameplay behaviors in the game. Using this assessment approach, the authors aim to prevent formalities in traditional testing so as to minimize test anxiety and construct-irrelevant variance. In Zoombinis, four CT components are measured alongside two gaming strategies: problem decomposition, pattern recognition, abstraction, and algorithmic thinking. Overall, this is an innovative assessment design and offers several advantages such as the collection of big data sets and inclusion of realworld problems and complexities. Evidence of reliability and validity have also been established 
through inter-rater reliability and convergent validity, suggesting the quality of this assessment approach.

\section{Towards an Integrative Approach in Measuring CT}

In general, assessments that target domain-specific CT skills serve the purpose of evaluating learners' knowledge and skills in programming environments or languages that are used in the classroom and are familiar to learners. This is ideal and sufficient when the goal of measuring CT is embedded into programming lessons within the computer science curriculum. However, if and when the objective is to capture the diversity of CT, solely using this type of assessment could limit the understanding of CT skills that are less programming-centric but more generalized to solving real-world problems. In this regard, Basso and colleagues [44] argue that solely using one type of assessment tool can lead to an incomprehensive view of CT skills. Doing so may also underrepresent important contexts in which CT could be manifested in, leading to issues of construct underrepresentation and validity issues. In particular, it might not serve the purpose of intervention studies, especially at the pre-test phase for students without any prior programming experience [27]. On the other hand, while domain-general assessments target cognitive and problem-solving skills, each of the tools focuses on a particular area. For example, the CTt measures programming concepts and Bebras ${ }^{\circledR}$ assesses general problem-solving. Hence, combining several assessment tools could be useful, such as the use of the lean assessment proposed by Wiebe et al. [27].

There is an emerging consensus amongst educators and researchers that the diversity of CT necessitates a broad range of content and contexts in assessment. For example, a recent study that explores teachers' perspectives regarding CT assessment suggests the importance of using various approaches or tools to measure CT comprehensively [19]. This perspective is not only shared amongst educators but also researchers. Grover [45] suggests the use of "systems of assessments" to assess both cognitive and non-cognitive aspects of CT. It is recommended that such an approach should include programming tasks (e.g., MCQs) and other measures (e.g., Parson's puzzles) [46]. Likewise, Kong [47] recommends using a combination of multiple-choice questions, programming projects, and survey instruments to cover the full spectrum of CT. Similarly, Román-González et al. [39] propose a comprehensive model of assessment that uses several validated tools to measure $\mathrm{CT}$ in intervention studies. All of these developments echo Huang \& Lool's [48] reflection that the usage of CT in one context is likely to transfer to other contexts, therefore they suggest researchers also consider other situations in which CT can also be evidenced outside of programming. In this regard, Basso and colleagues [44] proposed a comprehensive assessment approach that includes social-emotional aspects of CT development, including questionnaires and self-reports to measure coding performance and emotions, learning transfer, and cognitive as well as life skills.

An alternative approach, and perhaps a potentially integrated and psychometrically sound approach, is to expand the current scope of assessment design to encapsulate the breadth of CT by adopting a competency-based assessment approach. This integrative approach of assessment includes various assessment formats and sources so as to collect relevant evidence to support inferences about CT competency [49]. This integration could be derived from combining methods that assess multiple aspects of the competence as well as performance simultaneously [50]. In the following, CT competency is explained and a competency-based assessment is introduced and proposed.

\section{A COMPREHENSIVE ASSESSMENT OF CT COMPETENCY}

CT competency entails a set of cognitive skills that are useful for a broad range of computingrelevant tasks and contexts, including programming and, more broadly, problem-solving. That is, the dynamic combination of domain-specific skills and domain-general higher-order 


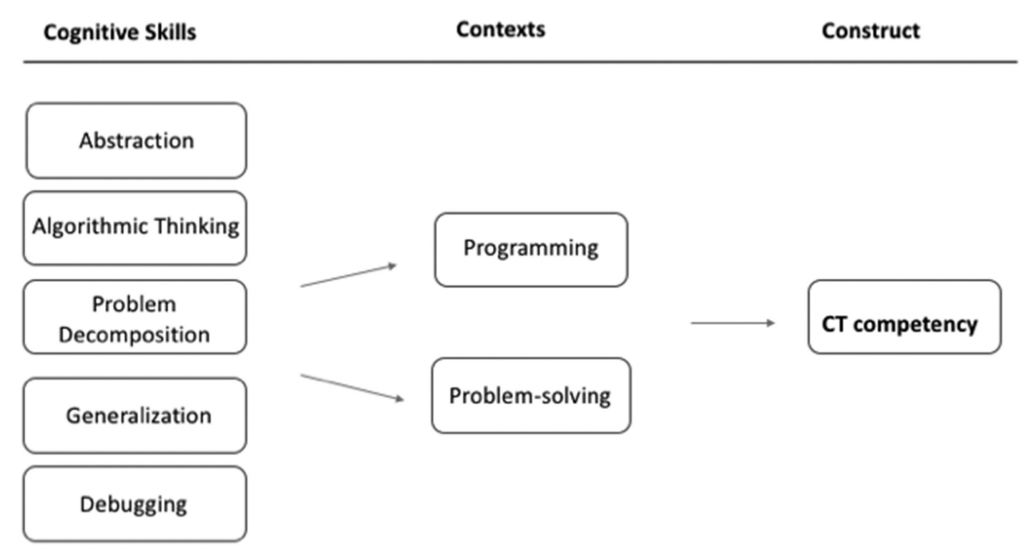

Fig. 1. A conceptual model of CT competency.

problem-solving skills $[19,51]$. These involve several theoretically recognized cognitive skills that are previously discussed: abstraction, algorithmic thinking, problem-decomposition, generalization, and debugging $[20,51,52]$. A conceptual model of CT competency is displayed in Figure 1.

With the increasing complexity of our environments and the world, these skills, when situated within the construct of CT competency, ought to meet the demands of everyday life. Hence, CT competency is less concerned about factual knowledge, but rather the utilization of skills, understanding, and knowledge of computing principles in diverse, and ideally, authentic situations. As such, it should be measured using a broad range of computing-relevant tasks that demand these skills in order to be reliably and validly measured in an assessment. Due to its complexity, it is not typically measured in a standard school assessment [43] and so far there has not been any measurement tool that assesses CT competency per se.

Based on the objective to measure CT competency, Computational Thinking Challenge (CTC), a computer-based assessment, was developed for upper secondary school learners. Its design recognizes that CT competency, like any other type of competency, cannot be validly measured by a single, isolated performance nor a single measurement method [53]. Given this, the assessment includes two contexts (programming and problem solving), two item formats, and three problem types. The goal is to encapsulate the aforementioned cognitive skills of CT in a more comprehensive manner. These skills are assumed to be used across all items regardless of context and format. Adopting an integrative approach, the assessment aims to extend the ways to which learners express their solutions, and hence allows a more extensive way for the demonstration of their competencies. The goals and design of the assessment are first explained. This is followed by an elaboration of the two problem types and formats of the items.

\section{Assessment Goals and Design}

Three goals underpin the design of the assessment: the measurement of CT competency, a hybrid approach of assessment, and a technology-enhanced assessment. First, CT competency supports real-world problem-solving abilities that are beyond the scope of programming proficiency [56, 16]. Based on Weinert's framework of competencies [57], CT competency is operationalized as a specialized cognitive competency that entails a set of mental prerequisites, contributing to performance in computing-relevant tasks. When measuring CT competency, solving complex and authentic real-world problems is essential. From an assessment perspective, measuring CT 
competency has several benefits: (1) it quantifies CT in a more neutral manner such that it is understood in a broader context and provides information on both programming and nonprogramming data; (2) it tests learners with novel real-world problems that are meaningful and pro-social, and; (3) it provides better integration with other disciplines (e.g., STEM) without the requirement of prior programming knowledge [27].

Second, the use of a hybrid approach recognizes that educational assessment could serve various purposes. In the context of the CTC, it integrates both functions of formative and summative assessments. The formative aspect includes feedback and thinking points in the form of an automated personalized report. The report aims to facilitate deeper learning strategies and metacognitive skills in CT development. Grover [46, 58] highlight that assessments do not necessarily function to grade students, but from a formative assessment approach they can improve teaching and learning, which is needed in CT assessments. The summative aspect provides concrete data to teachers and/or researchers regarding learners' overall performance. The key objective to this design was to serve the interests of the learners; to foster independent learning and self-regulation by providing immediate guidance and supportive feedback. As CT competency is not a subject on its own but a competency to develop overtime, it is vital that learners gain an understanding of their own capabilities and improvement areas.

Third, technology can greatly enhance the quality of educational assessment. Based on the wellestablished benefits of computerized assessments, the CTC was designed to be administered online. This increases time efficiency and scoring accuracy; it allows the integration of response time to capture processing speed and efficiency; it is compatible with interactive and immersive item design, and; it provides immediate delivery of personalized feedback to learners. Alves \& Hauck [26] illustrate that automatic assessment approaches allow the provision of real-time feedback, which improves programming competencies and enhances learning in computing education. The design aligns with The International Test Commission guidelines for Computer-Based and InternetDelivered Testing and the British Psychological Society guidelines for the development and use of computer-based assessment.

3.1.1 Problem Types of the Assessment. Problem Type 1: Parson's problems. Leveraging the computerized nature of the assessment, Parson's problems are used as a format to test programming proficiency. Parson's problems are also a useful formative learning and assessment activity for students to learn programming [56]. In this format, a generic language is used (i.e., move, turn, pick) such that it does not require prior knowledge in a specific language or syntax. These problems ask students to select the required fragments of code and rearrange them in the right order to formulate a correct solution in a fun and interactive way [59]. Before the respondents start, they are introduced to three commands: move (move the spaceship one step in the grid), turn (rotate the spaceship 90 degrees counterclockwise), and pick (pick up the astronaut). The mission of the task is to direct the spaceship to save the astronaut using the commands (see Figure 2).

This particular format allows the measurement of learners' CT competency in programming without their concern about making syntax errors. Cognitively, Parson's problems, as a code construction task, require a lower cognitive load because of the limited problem space compared to text- and blocked-based programming languages [60]. Previous studies have elaborated on the benefits of solving Parson's problems compared to code-writing: they are more sensitive to measuring students' learning gains [61,32]; more motivating and enjoyable to complete [59, 60, 62], and; increase students' future interest in computing activities [32]. Finally, aligning with the principle of fairness in educational assessment, the use of generic language is hoped to be accessible for students with diverse programming backgrounds and experiences. In so doing, it excludes any unnecessary barriers or construct irrelevance variance that may conceal conceptual understanding for some learners [43]. 
Snippets

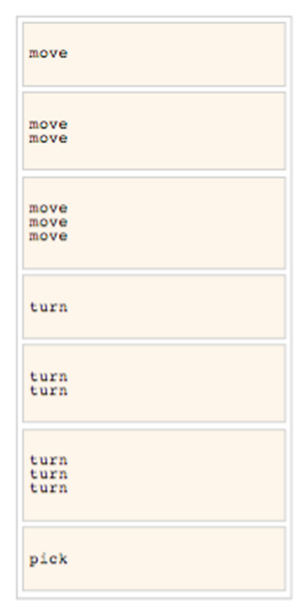

My Program

Drag the code snippets into the answer box in the correct order. require all of them!

Fig. 2. An example of Parson's problem.

Problem type 2: Multiple-choice question (MCQ). The same generic language (i.e., move, turn, pick) is used in the MCQs (Figure 3). The inclusion of this format was to ensure that the increased complexity of the algorithms being tested was not confounded with the details of the programming language itself. Grover [46] suggests well-designed MCQs can facilitate deeper understanding of programming. Although both formats are fundamentally designed to assess the same set of cognitive skills of CT in the programming context, each format allows students to express their solutions in a different manner. Parson's problems allow students to generate their solutions by selecting and rearranging the right sequence of code; MCQs focus on the selection of the most appropriate and efficient pre-formulated algorithm for a given question. Utilizing two different formats supports the measurement of $\mathrm{CT}$ competency in the programming context in a more comprehensive manner.

Problem type 3: Computational Problem-Solving Tasks. Enhancing higher-order problemsolving skills through the development of CT is a goal shared by educators. The ability to solve complex problems is a fundamental manifestation of learners' thinking and reasoning processes in $\mathrm{CT}$, which is beyond domain-specific knowledge and skills. Yet, problem-solving as a term is rather broad, and not all problems necessitate CT. According to Wing [63], the central focus should be on problem formulation in which a computational solution is admitted. Likewise, Kong [47] has recently proposed that problem formulation be included as a CT component. The idea of problem formulation is similar to the initial stage of problem-solving: to recognize and identify the problem at hand and to select the most efficient solution [64, 65]. The latter aspect, computational solution, calls for tasks that demand the aforementioned cognitive components. The tasks designed for this problem type target computational problem-solving skills in authentic real-world contexts with pro-social issues, such as solving an epidemiology issue. The items are answered in a multiplechoice format with four answer options and learners are asked to respond with the most efficient solution to a given problem (Figure 4).

\section{THE CURRENT STUDY}

An important consideration for a competency assessment regards appropriate problem contexts, types and item formats-that the diversity and breadth in coverage would not affect the robustness of the assessment. The overarching goal of this study is therefore to examine whether this 


\section{Epidemiology expert, please save the world!}

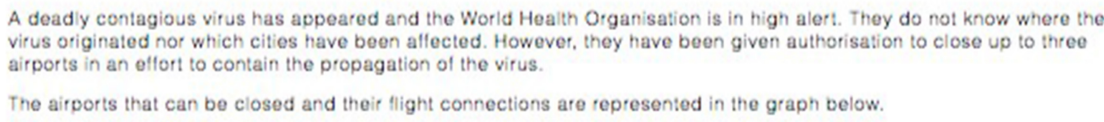

A deadly contagious virus has appeared and the World Health Organisation is in high alert. They do not know where the virus originated nor which cities have been affected. However, they have been given authorisation to close up to three airports in an effort to contain the propagation of the virus.

The airports that can be closed and their flight connections are represented in the graph below.

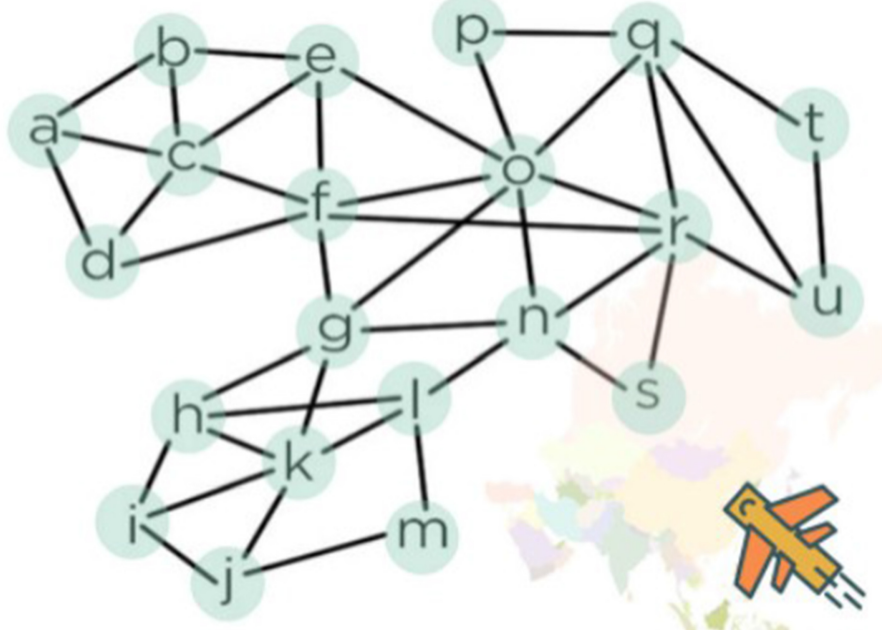

Which three airports would you close to ensure the smalles! possible disease propagation?
A) onr
B) fon
C) gor
D) $\circ g k$

Answer: Select an option $\checkmark$

Fig. 4. An example of problem-solving tasks.

the relationship between individual factors (e.g., programming experience and gender) and CT performance is also explored. In particular, the following research questions are investigated:

\section{Assessment quality:}

(1) Internal Consistency Reliability: Is the assessment internally consistent?

(2) Convergent Validity: Is CT competency associated with computer science performance?

\section{Psychometric properties:}

(3) Item fit: How valid are the items in assessing CT competency?

(4) Item difficulty: What is the range and the spread for the item difficulty parameter? What is the distribution for CT competency and item difficulty in this sample?

(5) Item/ Test Information: How reliable is each item and the whole assessment in estimating different CT competency levels? How much information does the assessment provide over the competency range?

\section{Programming Experience, Gender and CT Performance}

(6) Are individual factors associated with $\mathrm{CT}$ performance? 


\section{METHODS}

\section{Participants}

The sample was recruited from computer science classes in upper secondary schools in the United Kingdom. It was composed of a total of 119 students whose ages ranged from 14 to $19(M=16.11$; $S D=1.19)$. Except for six non-responses, $20.35 \%$ of the sample were year 9 students $(\mathrm{N}=23), 44.25 \%$ of them were year 10 students $(\mathrm{N}=50)$, and $35.40 \%$ of them were year 12 students $(\mathrm{N}=40)$. The majority of the sample were males $(81.5 \% ; \mathrm{N}=97)$. Most of the participants in this sample have had some coding/programming experiences prior to the study except for one non-response: $7.6 \%$ $(\mathrm{N}=9)$ with no experience, $1.7 \%(\mathrm{~N}=2)$ over one week, $5.9 \%(\mathrm{~N}=7)$ over one month, $35.3 \%(\mathrm{~N}=$ $42)$ over six months, $16 \%(\mathrm{~N}=19)$ over a year, $21 \%(\mathrm{~N}=25)$ over two years, and $11.8 \%(\mathrm{~N}=14)$ over three years.

\section{Materials and Procedures}

The study was approved by the Faculty of Education Ethics Committee at the University of Cambridge. Prior to this study, the assessment was internally reviewed. The assessment used in this study was the result of a three-stage small-scale pilot study. The first stage entailed a teacher survey regarding the important $\mathrm{CT}$ components to be measured. Teachers were asked about the core skills that $\mathrm{CT}$ entails and the results guided item design. The second phase was the administration of the assessment in its original paper-based version. The third stage was the comparison of the paper and computer-based versions. The reliability between the two versions was compared; the computer-based version was selected to be the final version based on its superior internal consistency reliability. The final version was then externally evaluated in an expert panel, judged by one assessment specialist from a UK exam board, one computer science education researcher, and one computer science education consultant of an education foundation.

The final assessment was administered online during computer science classes. The participants spent the allocated 51.45 minutes to complete 21 items (2.45 minutes per item). All items were scored dichotomously: correct (1) or incorrect (0). Participants had to answer each question in order to proceed to the next and they were unable to retrieve the previous question. Prior to the administration, computer science teachers received a study protocol, containing a list of standardized instructions, URL, and access code to the assessment. During the study, all participants were given a piece of paper and a pen to take notes when needed to prevent unintended testing consequences (e.g., cognitive load). Upon completion, each participant received a personalized feedback report. The participants' responses were automatically scored; the results were downloaded as a CSV file to be used, processed, and analyzed in R.

\section{Calculation and Research Questions}

The items were analyzed from a psychometric approach using the Rasch measurement model, a class of item response theory (IRT) model. Compared to the traditional classical test theory and related models that focus on a single sum score, a Rasch model could serve as a more appropriate means to evaluate CT competency. The rationales are three-fold. First, a competency is considered a disposition or a latent construct that cannot be directly observed. The latent nature of CT competency can be addressed by the Rasch model, which is a type of latent trait model. Second, IRT methodology has been suggested to be more robust for the development and validation of new competency and performance-based assessments [66]. Third, as an initial study of a new assessment, the Rasch model helps determine whether it covers an appropriate range of difficulty levels. This informs any necessary refinements or modifications of the items for future use. 
In this study, a dichotomous Rasch model was used to estimate item properties and characteristics by modeling item parameters at both the item level (i.e., probability of correct response) and test level [67]. Through this, respondents' latent CT competency (as denoted as $\theta$ from hereon) was also estimated. The Rasch model is specified below [56]:

$$
B_{n}-D_{j}=\operatorname{In}\left(P_{\mathrm{ni}} / 1-P_{\mathrm{ni}}\right)
$$

The relationship between a respondent's $\theta$ ( $\mathrm{B}_{\mathrm{n}}$; the level of CT competency) endorsing an item $\left(\mathrm{D}_{\mathrm{i}}\right.$; difficulty of the item) is expressed by the log of the respondent endorsing the item $\left(P_{\mathrm{ni}}\right)$ divided by the probability of the respondent not endorsing the item $\left(1-P_{\mathrm{ni}}\right)$.

Using this approach, other than reliability and validity, psychometric properties on item fit, item difficulty, and item/test information function were also estimated. Items that did not fit the criteria of these analyses need to be adjusted and revised so as to develop a refined and robust assessment. The analyses were conducted using the ltm [69], TAM [70], and mirt [71] packages in Rstudio.

The first research question concerns the reliability of the assessment. For this, internal consistency reliability was measured, referring to the extent to which items within an assessment measure various aspects of the same construct consistently [72]. In particular, Cronbach's alpha, Guttman's lambda 4 and 6, as well as person and item separation reliability statistics were examined. Cronbach's alpha measures internal consistency: the extent to which all the items in the CTC measure CT competency based on inter-relatedness of the items. As the alpha value could systematically underestimate reliability, other reliability indices such as the Guttman's lambda L4 and L6 were also investigated to provide a diverse perspective to reliability. L4 divides the items into two halves to calculate the covariance between the two halves, the L6 considers the variance of the errors [73]. On the other hand, person and item separation reliability statistics evaluate the reproducibility of the assessment and can be interpreted as the alpha value [74]. The former indicates the extent to which items distinguish respondents based on their $\theta$ location, the latter demonstrates how well the respondents can separate the items.

The second research question focuses on convergent validity, the extent to which the CTC is associated with self-reported computer science grades based on the latest classroom performance. Validity evidence was collected based on the relationships between the two domains and computer science performance. Moderate correlation coefficients were expected as an indication of acceptable convergent validity.

The third research question focuses on item fit. Amongst the many ways to infer validity, evaluating fit statistics is one way to provide evidence of construct validity. This was done by investigating the degree of suitability of the items in measuring CT competency. This process also elucidated the fit of the assessment- based on the items-to the Rasch model. To assess, the infit and outfit Mean Square Statistics (MNSQ) were computed for each item. Infit (weighted fit statistics) is based on the information-weight sum and is sensitive to unexpected responses to items that are close to the respondents' $\theta$ level. Outfit (unweighted standardized fit statistic) is based on the mean of squared standardized residuals and is sensitive to unexpected responses on items that are extreme for respondents' $\theta$ level [75]. The critical values for MNSQ have been suggested to range between $0.5-1.5$ logits; items that deviated from this range would be considered misfits and may suggest a violation of unidimensionality and validity [74].

The fourth research question concerns item difficulty. For this, the $b$-parameter was inspected, informing the location at which a respondent had a $50 \%$ probability of endorsing an item. Easy items have lower $b$ values than difficult items. Items should be within the proposed range of -3 to +3 logits to reflect a well-balanced difficulty level [78]. To investigate the spread of item difficulty, an item characteristic curve (ICC) was generated to represent the probability of a correct response as a function of $\theta$. Also, a Wright map (person-item map) was presented to 
graphically illustrate the respondents' $\theta$ and estimated item difficulties on the same logit scale. The map reveals how well the item difficulties are against the respondents' $\theta$. The left side of the map represents respondents' $\theta$ and the right side represents the items. The highest $\theta$ level and the most difficult items are displayed on top of the map; the lowest $\theta$ level and easiest items are on the bottom. When the $\theta$ location is above the item location, it indicates the probability of endorsing a particular item is higher than $50 \%$.

The fifth research question focuses on item/ test information. Reliability indices are often not based on the assumption that reliability can be influenced by respondents' $\theta$ and item parameters. This raises the question regarding which $\theta$ group (low, average, or high performing group) is most reliably measured by each item and the overall assessment. To address this issue, the Item Information Functions (IIF) and Test Information Function (TIF) were examined. IFF shows the amount of information an item provides with the greatest precision for a specific $\theta$ level in relation to the item's difficulty parameter. The sum of IIF makes up the TIF which signifies how well each $\theta$ level is being estimated by the overall assessment. Both functions inform how well the assessment is in estimating $\theta$ over the wide range of $\theta$ levels.

The sixth and last research question explores individual factors that have been shown in previous studies to associate with CT performance. In particular, the interrelations between programming experience, gender, and CT performance were investigated using the Spearman's rho correlation and independent samples t-test.

\section{RESULTS}

\section{Descriptive Statistics and Correlations}

Descriptive statistics are presented in Table 2. A correlation matrix is displayed in Table 3, illustrating the correlation coefficients between the two domains and three problem types with the total test score. The relationships between all variables are significant and strong. Regardless of the different contexts and formats, they are all associated with the total test score.

\section{Assessment Quality}

6.2.1 Internal Consistency Reliability. Internal consistency reliability analysis was conducted on the 21 items of the CTC using Cronbach's alpha. Based on the criteria of alpha proposed by [76], the CTC demonstrated good reliability, $\alpha=.71$. Additionally, Guttman's lambda 4 (L4; betweenscore correlation) and 6 (L6; squared multiple correlation) were computed. Similar to the alpha value, the results indicated adequate reliability, $\mathrm{L} 4=.82$ and $\mathrm{L} 6=.75$. As part of the main analysis, person and item separation reliability statistics were also calculated. The estimated person reliability coefficient in this sample was .70 and the item separation reliability was .68. Both suggest sufficient sensitivity in distinguishing between various performing groups [77].

6.2.2 Convergent Validity. A correlation matrix is displayed in Table 4, presenting the correlation coefficients between the two contexts (programming and problem-solving), the total test score of the assessment, and computer science grades. Overall, convergent validity was evidenced by the significant and positive relationship between the overall test performance and grades $(\mathrm{r}=.48, p<.001)$. The relationship between problem-solving and CS grades was slightly stronger $(\mathrm{r}=.45, p<.001)$ than programming $(\mathrm{r}=.40, p<.001)$.

\section{Psychometric Properties: A Rasch Model Analysis}

Prior to conducting the Rasch model, the assumption of unidimensionality was tested using the Martin-Löf test in R studio. The chi-square test was non-significant $(p=.76)$, indicating the hypothesis of unidimensionality should not be rejected. 
Table 2. Descriptive Statistics

\begin{tabular}{|c|c|c|c|c|c|}
\hline Context & Problem Type & Item & $\mathbf{M}$ & SD & Accuracy \\
\hline \multirow[t]{9}{*}{ Programming } & Parson's problems & Q1 & .77 & .42 & $77 \%$ \\
\hline & & Q2 & .58 & .49 & $58 \%$ \\
\hline & & Q3 & .34 & .47 & $34 \%$ \\
\hline & & Q4 & .38 & .49 & $38 \%$ \\
\hline & MCQs & Q5 & .27 & .44 & $27 \%$ \\
\hline & & Q6 & .43 & .50 & $43 \%$ \\
\hline & & Q7 & .20 & .40 & $20 \%$ \\
\hline & & Q8 & .33 & .47 & $33 \%$ \\
\hline & & Q9 & .61 & .49 & $60 \%$ \\
\hline \multirow[t]{13}{*}{ Problem-solving } & Problem-Solving & Q10 & .29 & .46 & $29 \%$ \\
\hline & MCQs & Q11 & .28 & .45 & $28 \%$ \\
\hline & & Q12 & .29 & .45 & $29 \%$ \\
\hline & & Q13 & .07 & .27 & $8 \%$ \\
\hline & & Q14 & .31 & .46 & $31 \%$ \\
\hline & & Q15 & .15 & .36 & $15 \%$ \\
\hline & & Q16 & .53 & .50 & $53 \%$ \\
\hline & & Q17 & .26 & .44 & $26 \%$ \\
\hline & & Q18 & .90 & .30 & $90 \%$ \\
\hline & & Q19 & .34 & .47 & $34 \%$ \\
\hline & & Q20 & .11 & .31 & $11 \%$ \\
\hline & & Q21 & .31 & .46 & $31 \%$ \\
\hline & & Total & 7.74 & 3.55 & \\
\hline
\end{tabular}

Table 3. Pearson Correlation Matric

\begin{tabular}{cc}
\hline & Pearson's r \\
\hline Total score - Programming & $.88^{* * *}$ \\
Parson's problems & $.70^{* * *}$ \\
Programming MCQs & $.72^{* * *}$ \\
Total score - Problem-solving & $.88^{* * *}$ \\
Programming - Problem-solving & $.55^{* * *}$ \\
\hline Notes. ${ }^{*} p<.05,{ }^{* *} p<.01,{ }^{* * *} p<.001$. &
\end{tabular}

6.3.1 Item Fit. Table 5 presents the results of the fit statistics. The infit values ranged from $0.92-$ 1.11 logits; the outfit values ranged from 0.81-1.41 logits. As shown, all the items were within the acceptable range of 0.5-1.5 logits [61]. An examination of the corresponding t-statistics ( $\mathrm{z}-$ standard) also confirmed that both the infit and outfit values were within the proposed range of -2 to +2 logits [78]. That is, none of the items were significantly underfitting (too much variation) or overfitting (too little variation). This indicates that each item was consistent with the way the respondents responded to other items in the assessment. Overall, the results demonstrated a good fit to the model and provide supporting evidence of construct validity.

6.3.2 Item Difficulty. Overall, the results suggested that the items covered a wide and acceptable range of item difficulty spectrum (see Table 5). The $b$ values of all 21 items ranged from -2.44 (90\%; item 18; lowest difficulty level) to 2.78 ( $8 \%$; item 13 ; highest difficulty level) logits, and the majority of them demonstrated medium difficulty levels ( $0-1.5$ logits). Parson's problems ranged from -1.40 
Table 4. Pearson Correlation Matrix of CT Performance and CS Grades

\begin{tabular}{lc}
\hline & Pearson's r \\
\hline Total score - CS grades & $.48^{* * *}$ \\
Programming score - CS grades & $.40^{* * *}$ \\
Problem-solving score - CS grade & $.45^{* * *}$ \\
\hline
\end{tabular}

Notes. ${ }^{*} p<.05,{ }^{* *} p<.01,{ }^{* * *} p<.001$.

Table 5. Item Parameter Estimation and Fit Statistics

\begin{tabular}{|c|c|c|c|c|c|c|c|c|c|c|}
\hline Context & Problem Type & Item & Difficulty & $\mathrm{SE}$ & Outfit & $\mathbf{t}$ & $p$ & Infit & $t$ & $p$ \\
\hline \multirow[t]{9}{*}{ Programming } & Parson's Problems & Q1 & -1.40 & 0.23 & 0.85 & -1.26 & 0.21 & 0.97 & -0.26 & 0.79 \\
\hline & & Q2 & -0.37 & 0.20 & 0.87 & -1.91 & 0.06 & 0.90 & -1.51 & 0.13 \\
\hline & & Q3 & 0.78 & 0.21 & 0.90 & -1.26 & 0.21 & 0.92 & -0.95 & 0.34 \\
\hline & & Q4 & 0.57 & 0.20 & 0.94 & -0.88 & 0.38 & 0.95 & -0.66 & 0.51 \\
\hline & MCQs & Q5 & 1.14 & 0.22 & 0.94 & -0.59 & 0.55 & 0.96 & -0.37 & 0.71 \\
\hline & & Q6 & 0.33 & 0.20 & 0.92 & -1.26 & 0.21 & 0.93 & -1.00 & 0.32 \\
\hline & & Q7 & 1.56 & 0.24 & 1.09 & 0.63 & 0.53 & 1.00 & 0.07 & 0.94 \\
\hline & & Q8 & 0.82 & 0.21 & 0.92 & -0.95 & 0.34 & 0.93 & -0.84 & 0.40 \\
\hline & & Q9 & -0.49 & 0.20 & 1.14 & 1.89 & 0.06 & 1.09 & 1.17 & 0.24 \\
\hline \multirow[t]{12}{*}{ Problem-solving } & Problem-Solving & Q10 & 1.00 & 0.21 & 1.05 & 0.55 & 0.58 & 1.02 & 0.25 & 0.80 \\
\hline & MCQs & Q11 & 1.09 & 0.22 & 0.95 & -0.49 & 0.63 & 0.98 & -0.15 & 0.88 \\
\hline & & Q12 & 1.05 & 0.22 & 0.96 & -0.41 & 0.68 & 0.98 & -0.2 & 0.84 \\
\hline & & Q13 & 2.78 & 0.36 & 1.13 & 0.47 & 0.64 & 1.03 & 0.2 & 0.84 \\
\hline & & Q14 & 0.91 & 0.21 & 1.04 & 0.43 & 0.67 & 1.03 & 0.37 & 0.71 \\
\hline & & Q15 & 1.94 & 0.27 & 1.29 & 1.59 & 0.11 & 1.09 & 0.57 & 0.57 \\
\hline & & Q16 & -0.14 & 0.20 & 0.97 & -0.40 & 0.69 & 0.99 & -0.18 & 0.85 \\
\hline & & $\widetilde{Q} 17$ & 1.19 & 0.22 & 1.18 & 1.62 & 0.11 & 1.11 & 1.02 & 0.31 \\
\hline & & Q18 & -2.44 & 0.31 & 0.81 & -0.83 & 0.41 & 0.99 & 0.01 & 0.99 \\
\hline & & Q19 & 0.78 & 0.21 & 1.14 & 1.58 & 0.11 & 1.09 & 1.05 & 0.30 \\
\hline & & Q20 & 2.35 & 0.30 & 1.41 & 1.71 & 0.09 & 1.08 & 0.45 & 0.66 \\
\hline & & Q21 & 0.91 & 0.21 & 0.97 & -0.37 & 0.71 & 1.00 & 0.06 & 0.95 \\
\hline
\end{tabular}

Note. $\mathrm{SE}=$ standard errors; Outfit/Infit = item outfit statistics (values between $0.5-1.5$ are acceptable); $\mathrm{t}=\mathrm{t}$ value for the fit statistics (values between -2 and +2 are acceptable); $p=p$ value for outfit statistics (significant results may indicate misfit).

to 0.79 logits (38-77\%); MCQ programming problems ranged from -0.49 to 1.56 logits $(20-61 \%)$, and; problem-solving tasks ranged from -2.44 to 2.78 logits (8-90\%). The easiest and most difficult items were part of the problem-solving tasks. All the items were within the proposed range of -3 to +3 logits, indicating a well-balanced difficulty level [68]. This is evidenced by the widespread and little overlaps between items in terms of their difficulty levels.

Figure 5 presents the ICC which shows the spread of the item difficulties. As illustrated, the probability of endorsing item 18 , the easiest item, was approximately $90 \%$ for respondents with an average $\theta$ level. It was answered correctly by most respondents on the lower $\theta$ location. However, the probability of endorsing item 13 , the most difficult item, was approximately $8 \%$. Only those who were at a higher $\theta$ level answered this item correctly.

All the items appeared to fit the monotonicity assumption of item quality: as $\theta$ increased the probability of respondents endorsing an item also increased. 


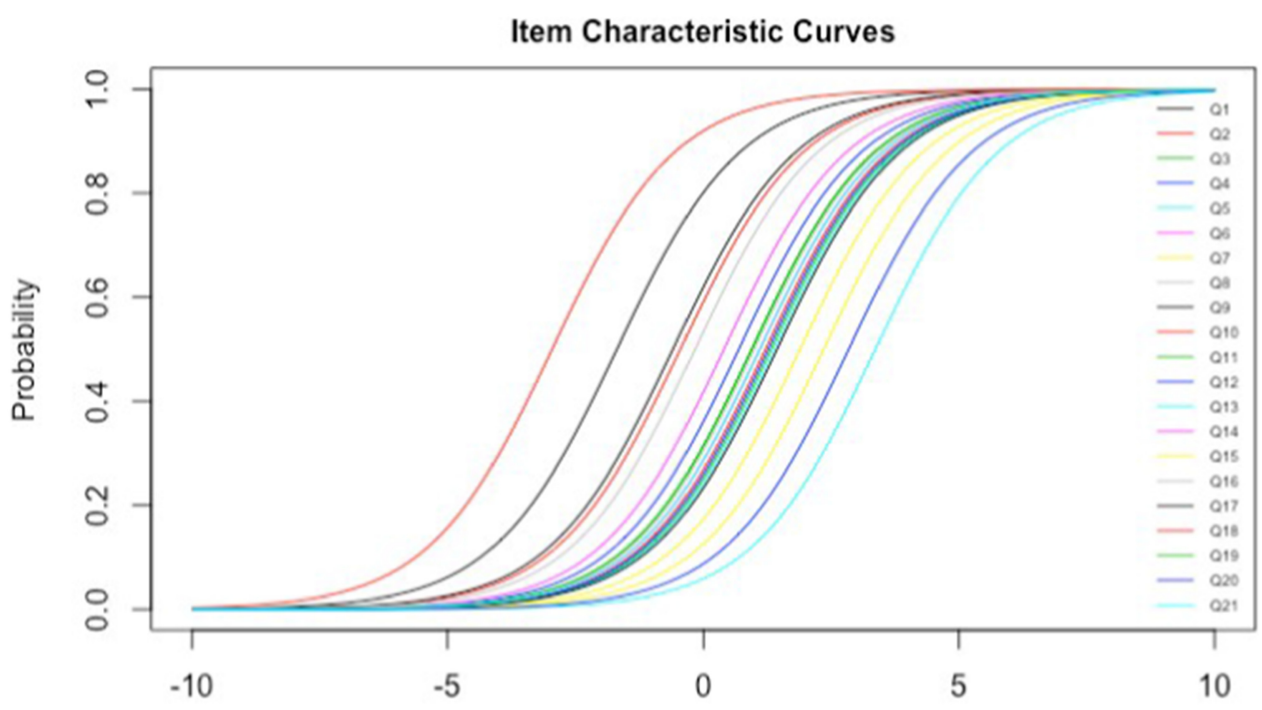

\begin{abstract}
Ability
Fig. 5. Item Characteristics Curves of the 21 items of CTC. Notes: Items on the upper left (i.e., Q18, Q1 and Q 9) represent easy items as the probability of correct response is high for low-performing respondents. The curves in the center (i.e., Q3, Q19 and Q8) represent items of medium item difficulty. The lower right curves (i.e., Q13, Q20 and Q15) represent difficult items; the probability of a correct response is low for most respondents and only increases when a higher level of performance is reached.
\end{abstract}

Figure 6 displays the Wright map (person-item map). The map presents the distribution of respondents' $\theta(M=.00, S D=.98)$ and the estimated item difficulty $(M=.68, S D=1.18)$. As suggested in the map, items 13,20 , and 15 were only endorsed by those located at the top (+2 logits) of the $\theta$ distribution while item 18 was endorsed by almost all the respondents except for the very few who were located at the bottom of the $\theta$ distribution (below -2 logits). In the case of this assessment, the items were slightly challenging for the respondents with an average $\theta$.

6.3.3 Item and Test Information. The item information curves (IIC) are displayed in Figure 7. It illustrates that the majority of the items were widely spread and covered a range of $\theta$ levels, with the majority of the items clustered around the center on the $\theta$ scale. According to the test information curve (TIC) displayed in Figure 8, the CTC provided precise information for $\theta$ levels in the interval between -4 and 4 , capturing $86.42 \%$ of the respondents. Thus, the items were reliable for the majority of the respondents, albeit its greatest precision (the peak of the curve) was at the interval between 0 and 2, capturing $32.51 \%$ of the respondents with average to slightly above average $\theta$ levels. Interpreting this against the Wright map, the results further suggested that item difficulty was slightly above the respondents' $\theta$ levels. Nonetheless, it is ideal that the test function has high information with $\theta$ level ranged from -4 to 4 such that most of the respondents could be estimated precisely.

\title{
Programming Experience, Gender, and CT Performance
}

Descriptive statistics were used to explore the variables in programming experience, gender, and CT performance (see Table 6). Performance distribution of students with different programming backgrounds was investigated using ggplot2 in R Studio [79]. Students' programming experience 


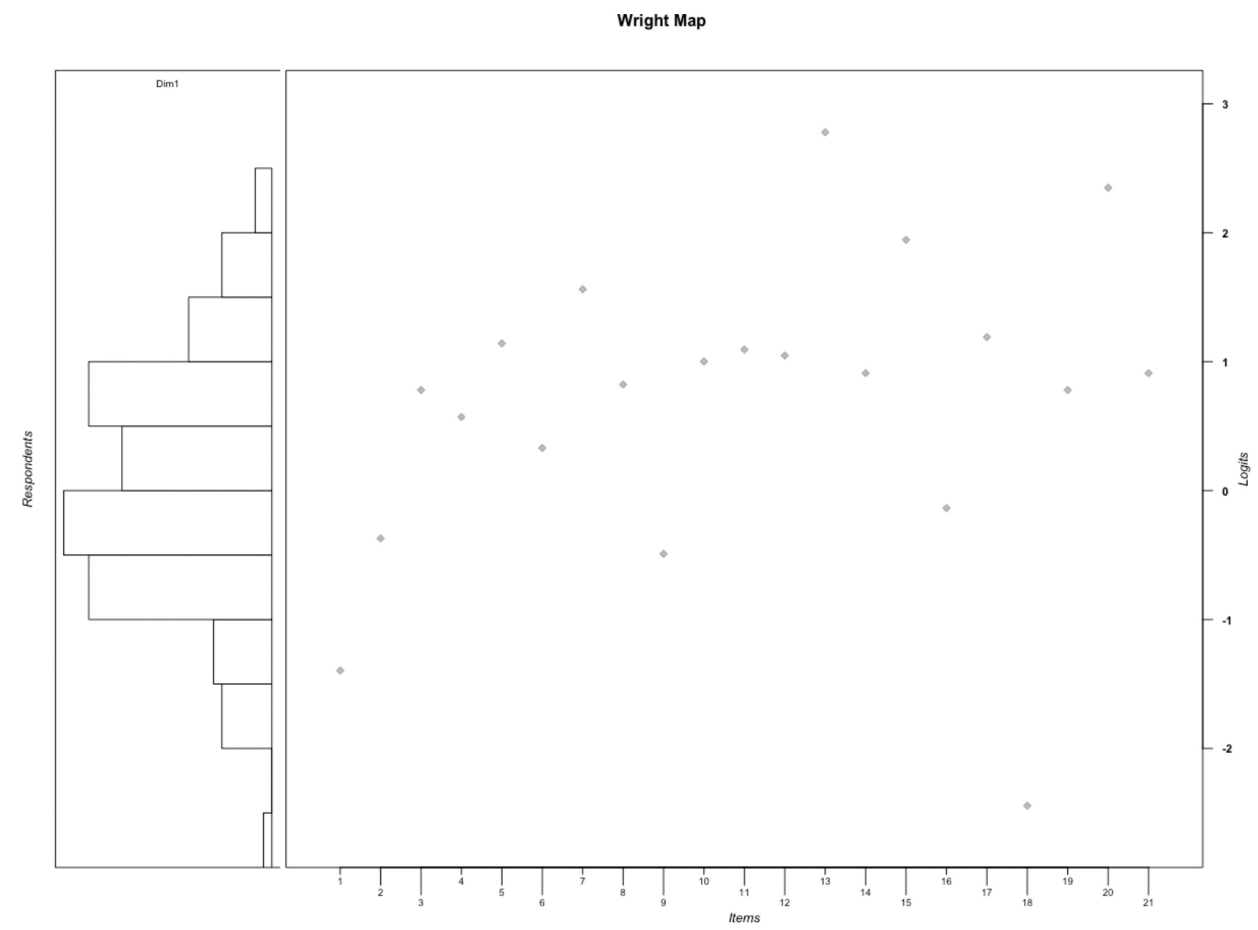

Fig. 6. A Wright map of person and item distribution. Notes. Left column represents respondent $\theta$; the right column represents item difficulty.

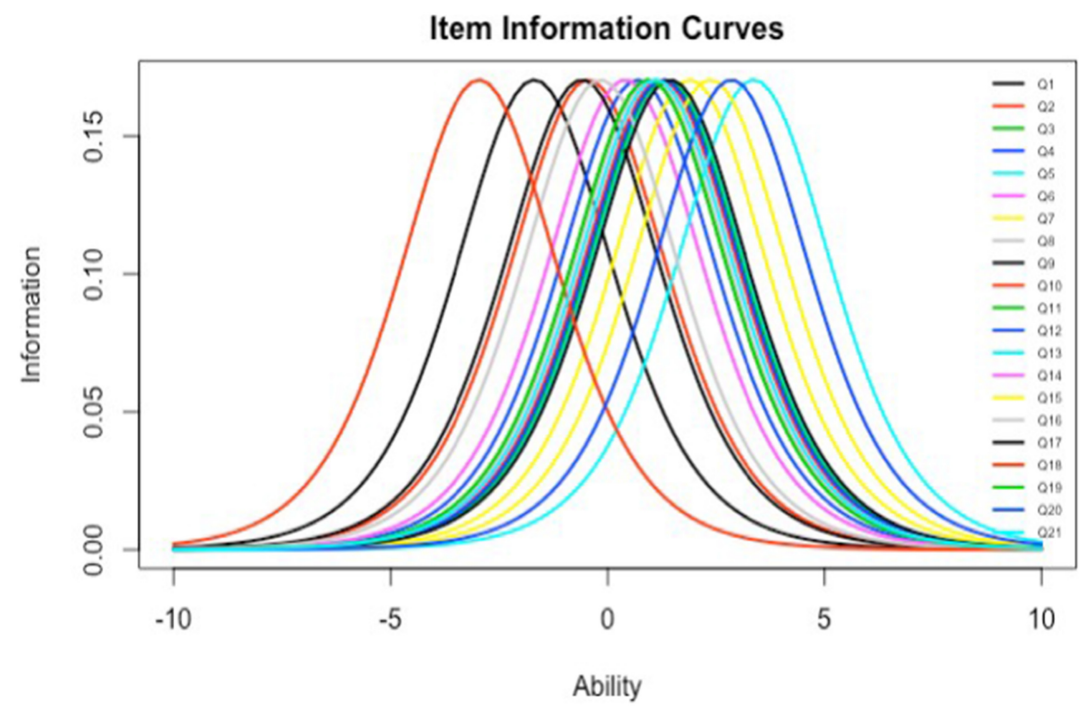

Fig. 7. Item Information Curves. 


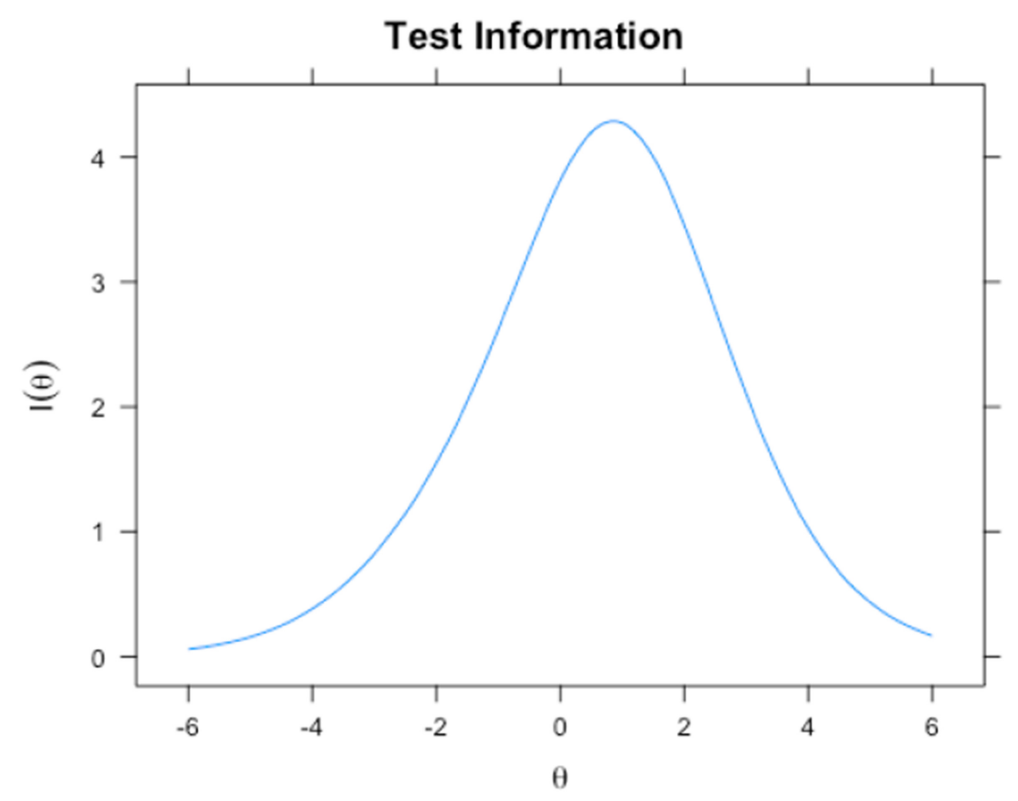

Fig. 8. Test Information Curve.

Table 6. Descriptive Statistics

\begin{tabular}{lcccc}
\hline \multicolumn{5}{c}{ Total } \\
\hline & Novice & Intermediate & Proficient & Advanced \\
\hline $\boldsymbol{N}$ & 9 & 51 & 19 & 39 \\
Mean & 5.33 & 7.06 & 7.05 & 9,51 \\
SD & 2.69 & 3.27 & 2.92 & 3.74 \\
\hline
\end{tabular}

was categorized into four larger groups except for non-responses: Novice (no prior experience; $\mathrm{N}=9$ ), Intermediate (6 months or less; $\mathrm{N}=51$ ), Proficient (1 year; $\mathrm{N}=19)$ and Advanced (2-3 years; $\mathrm{N}=39$ ). The performance distribution for each group is illustrated in Figure 9. As shown, the advanced group obtained the highest scores $(M=9.51, S D=3.74)$. Lastly, gender was included in a scatterplot to further examine if there was a gender difference; the results are displayed in Figure 10.

Spearman's rho correlation was then conducted. The overall results indicated that there was a significant moderate association between programming experience and overall CT performance, $\left(\mathrm{r}_{\mathrm{s}}(119)=.34, p<.001\right)$. However, by item formats, students' programming background was only related to Parson's problems $\left(\mathrm{r}_{\mathrm{s}}(119)=.34, p<.001\right)$ and problem-solving tasks $\left(\mathrm{r}_{\mathrm{s}}(119)=.26\right.$, $p<.001)$ but not programming MCQs $\left(\mathrm{r}_{\mathrm{s}}(119)=.14, p=.12\right)$.

The gender difference suggested in Figure 10 was further examined. An independent samples t-test was conducted to compare the overall CT performance between male and female students. The results indicated that there was a significant difference in performance between male students $(M=8.15, S D=3.52)$ and female students $(M=5.88, S D=3.12), t(112)=2.49, p<.05$. The effect size for this analysis was found to be medium, Cohen's $d=.68$. A Spearman's rho correlation was then conducted for further investigations. The results suggested that while there was a significant association between programming experience and overall $\mathrm{CT}$ performance, $\left(\mathrm{r}_{\mathrm{s}}(97)=.43, p<.001\right)$ for male students, there was not for female students $\left(\mathrm{r}_{\mathrm{s}}(17)=.10, p=.71\right)$. 


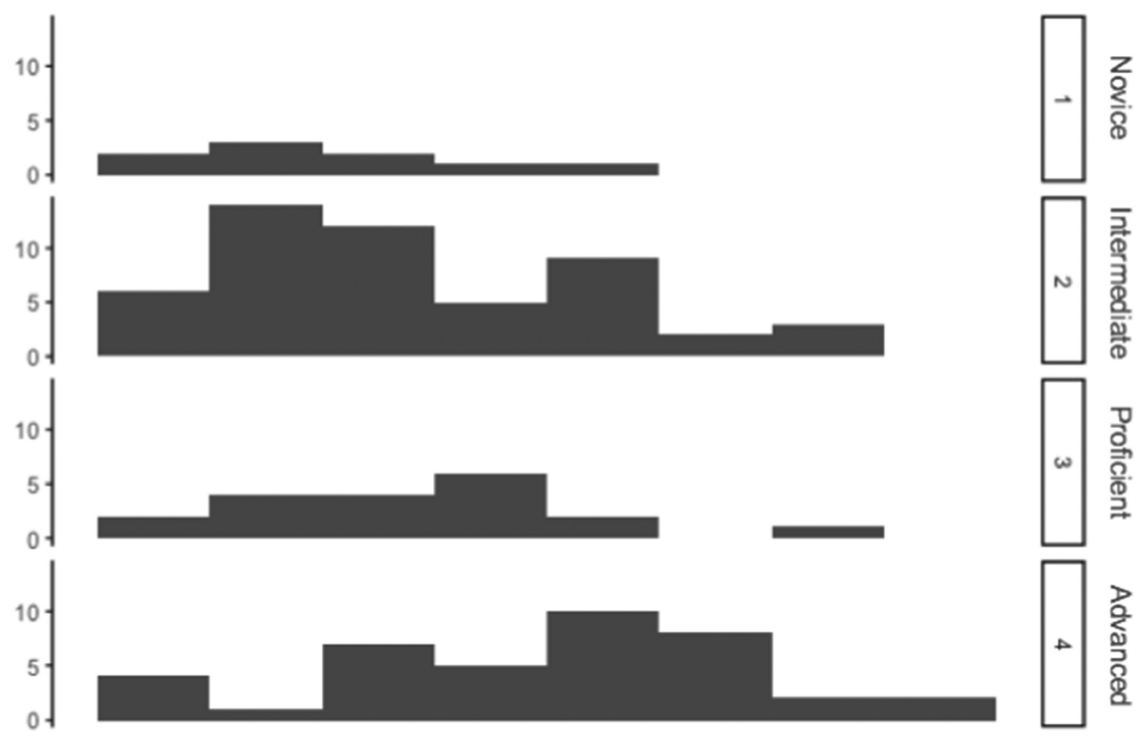

Total score

Fig. 9. Performance distribution of different programming groups.

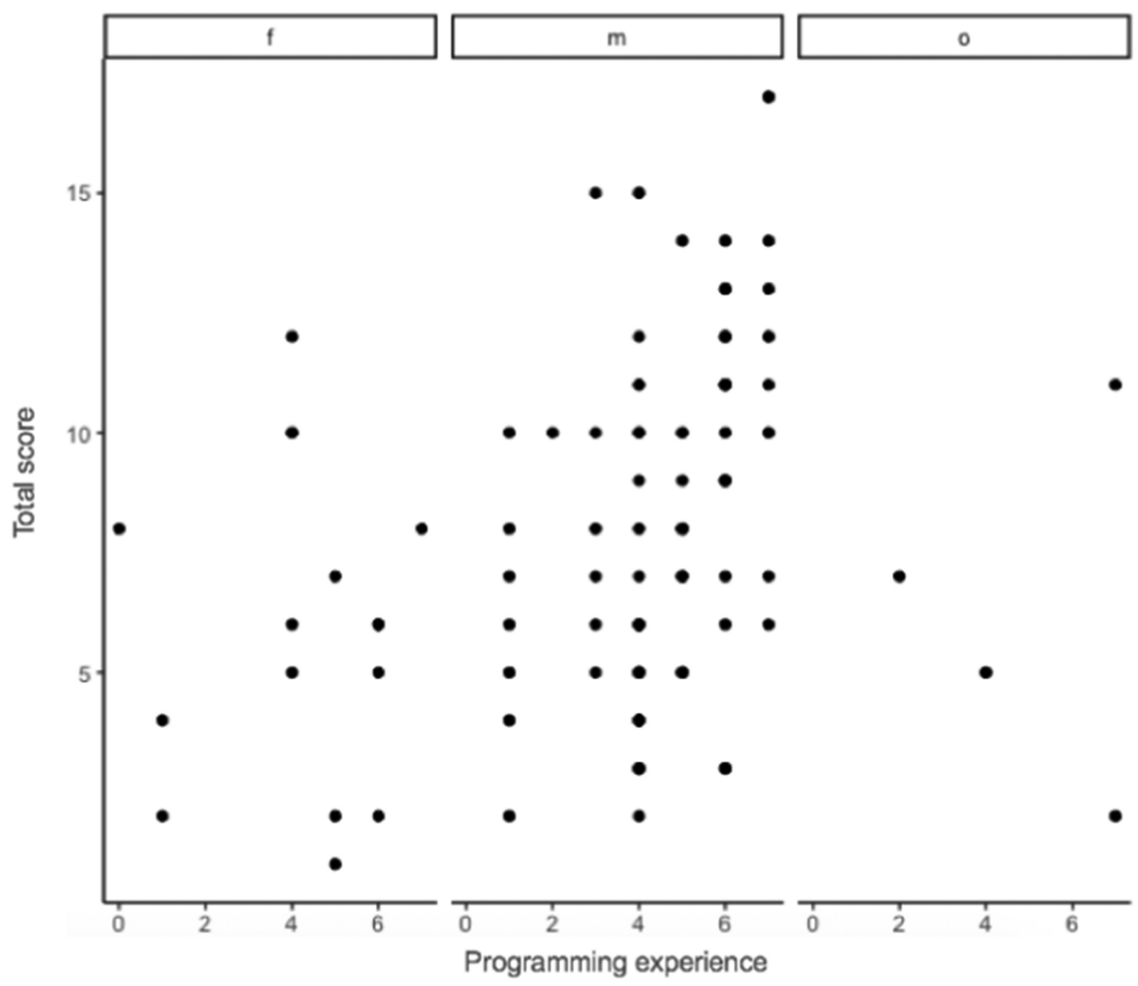

Fig. 10. Scatterplot of gender, programming experience, and total score. 


\section{DISCUSSION}

$\mathrm{CT}$, as a competency, is an emerging view in the current literature $[3,56]$. Assessing CT competency has a significant potential to help better understand its application in diverse problems across disciplines. Indeed, there is a growing consensus amongst educators and researchers to assess CT using multiple approaches to avoid the limited scope in understanding its development $[19,39,44$, 47]. For example, Grover [45] recommends the use of multiple but complementary measurement tools to measure CT in a more comprehensive manner. However, challenges arise when the nature of competence requires the integration of multiple contexts, problem types, and item formats that necessitate the manifestation of $\mathrm{CT}$ competency. Extending and continuing the work pioneered by others in addressing this issue $[3,39,45]$ as well as corresponding to the necessity for well-validated assessment tools $[1,36,39]$, this is one of the first studies to develop and validate a CT competency assessment through rigorous analytical techniques. In so doing, it suggests an integrated assessment approach could be a promising strategy to evidence CT development holistically.

\section{Quality of Assessment: Internal Consistency Reliability and Convergent Validity}

One of the indicators of a robust assessment is reliability. In this study, several reliability analyses were conducted to provide a comprehensive view of reliability. Results of the alpha, as well as lambda 4 and 6, suggest that despite various domains, problem types, and formats used in the assessment, items can reliably measure CT competency. Moreover, the results in the person and item separation reliability statistics of the Rasch model helped evaluate the extent of success in assessment development and in monitoring its utility in the future [80]. The overall results in the person separation statistics demonstrated sensitivity of the items in distinguishing different performing groups. That is, the sample ability variance was adequate due to the wide ability range captured and distinguished by the items in the assessment. This finding is crucial, particularly due to the diverse programming experience in this sample, which could be interpreted alongside the last research question and related findings. Aside from the person separation reliability statistics, items ought to be well separated in difficulty in order to have meaningful interpretations of the construct. The results from item separation statistics indicated a wide difficulty range represented by the item difficulty variance. Overall, the results are promising and suggest the feasibility of a comprehensive assessment in measuring CT competency in a reliable manner.

Another indicator of a quality assessment is validity. The significant association between the overall assessment performance and CS grades provided evidence to support convergent validity. Hence, the theoretical assumption that CT competency and CS are related is supported albeit the strength of the relationship is moderate. This is reasonable and expected as convergent validity focuses on the relationship between related constructs rather than the same construct. The variances not explained in this relationship could be due to both practical, methodological, and theoretical factors. Practically, several elements in the assessment are not straightly curriculum-based, such as the use of a generic language (rather than a visual programming language or a standard language taught at school), the inclusion of Parsons' problems, and the unfamiliar problem contexts to solve programming questions. In this regard, future studies can explore the possibility of using this assessment as a tool to measure learning transfer. Methodologically, a more appropriate measurement is a task that also combines aspects of programming and problem-solving such as the computational thinking practices test [36] rather than CS grades. Lastly and theoretically, the relationship between CT competency and CS grades might not be linear or co-exist concurrently (which would have reflected a stronger correlation): ability in CS could arguably precede CT development. This could possibly be the case given the results in Section 6.4. Nonetheless, as both contexts in the assessment were related to CS performance, teachers and researchers can 
customize its utility: choosing to use both contexts and all three problem types in a single setting or to select specific items according to specific learning goals or research purposes.

\section{Psychometric Properties}

As a new assessment, psychometric properties at the item level were explored for future refinement and development. Based on the statistics on item fit and difficulty, none of the items appeared to be problematic and are well within the range for a productive assessment. The findings suggest that the 21 items have achieved the required and acceptable range of difficulty levels: none of the items were extremely easy nor difficult. Amongst all formats, problem-solving tasks had the widest spread in terms of difficulty level and accuracy rate (8\%-90\%), indicating more power in distinguishing different performing groups. However, the three most difficult items belonged to this format $(\mathrm{Q} 13,15,20)$, turning to the need for teachers to pay more emphasis on generic problem-solving skills in non-contextual problems. In the programming context, compared to programming MCQs $(20 \%-61 \%)$, performance on Parson's problems yielded a better accuracy rate $(34 \%-77 \%)$, reinforcing the positive findings in previous studies and suggesting the promise of using Parson's problem in future assessment design. Interpreting these results alongside the internal-consistent reliability, items from different formats appeared to complement each other in measuring CT competency. This is useful for future theory development regarding CT competency, particularly in examining whether the construct can be explained by and constitute both programming and problem-solving CT skills. This is a promising direction for future studies as a theoretical account of CT competency is still scarce in the current literature.

Overall, with the wide range of item difficulty, the items appeared to have a good variability and were suitable to be used by learners with various $\theta$ levels. The respondents' $\theta$ also appeared fairly uniform and normally distributed, suggesting there wasn't any floor or ceiling effect. However, the distributions on the Wright map and the TIC suggest that learners who perform slightly above average might be best suited for the assessment. For this reason, the assessment may serve as a tool to help distinguish groups of performers. Together, these results provide supporting evidence to the quality of the items and suggest the appropriateness of using it to measure learners with different ability levels.

\section{Programming Experience, Gender, and CT Performance}

Performance is not solely dependent on objective psychometric properties but also subjective and personal factors. For example, the positive influence of programming experience on CT has been noted [81] and some evidence indeed suggests prior programming experience can impact CT development [29]. In this sample, the highest performing group was students who have an advanced background in programming (2-3 years). However, the underlying mechanisms of the relationship between programming background and CT competency are less known. The findings in this study suggested that the relationship between students' programming background and CT performance is less straightforward than expected: item format and gender could potentially play a role. Upon a more in-depth analysis, the results indicated that this association depends on item format. Whereas programming background was linked to Parson's problems and problem-solving tasks, it was not associated with the more traditional item format of programming MCQs. Although this finding is counterintuitive, this illustrates the potential to use the latter format with students with no prior to little programming experience and the former two for more advanced students. On the other hand, there was a significant gender effect in performance and that programming background only impacted male students and not female students. The results align with the findings in [17, 84], which reveal larger gender difference for students in $8^{\text {th }}$ grade and above. It should be noted, however, that the sample size for male and female students was unequal in this study so any 
interpretations should be made cautiously. Nonetheless, other personal factors, such as motivation, engagement, identity [82, 83], should be incorporated in future studies to further understand predictors of CT competency amongst male and female students. Such findings can shed light to better assessment design and contribute to the emerging area of gender differences in computing education.

\section{Limitations and Suggestions}

Several additional limitations ought to be addressed when interpreting the above results. As an initial study of the assessment, the sample size restricted the complexities allowed in a model and hence the ability to estimate other important item parameters. In the future, a large sample of respondents is required in order to accurately estimate other IRT item parameters (e.g., discrimination) as well as to compare goodness-of-fit between different models. When such parameters are analyzed, redundant items based on low discriminative power could then be eliminated to achieve a shorter and more efficient version of the assessment. Moreover, a larger sample size provides a stronger statistical power to test multidimensional IRT models, which might be more theoretically sensible and suitable for competency-based assessments such as the CTC. Beyond the sample size, an expansion of the item bank could be equally valuable as it allows for the development of a computer adaptive test for CT, which could be more precise and accurate than the current fixed version. For example, Hogenboom et al. [85] have demonstrated the benefits of this assessment approach in measuring programming concepts in younger children-this could be extended to CT and to older children.

A key factor in assessment quality is validity and several factors could have potentially threatened validity in this study. For example, the sample was narrowed to only students in the UK and consisted of more male students than female students. This is not representative for high school students in general and thus generalization is difficult to achieve. The issue of representativeness has remained to be a concern in computer science education research and community, as highlighted by The Royal Society [19]. In particular, gender diversity is considered a problematic issue in enrollment, and this is an implicit limitation of this study to note.

Related to threats to validity is the finding that programming experience impacted male students' performance. While having a sample with diverse programming experience enhanced representativeness, the finding could infer this as a confounding variable for male students. Future research could control for prior programming experience when using this assessment so as to disentangle the effects between programming proficiency and CT competency. Another means to address this issue is to evaluate the differential item functioning in a larger sample. This would allow the examination of bias in person measurement across gender, ethnicity, and background as measurement invariance could play a role. In so doing, it helps address the question of whether item responses are systematically influenced by other personal factors.

Lastly, as the establishment of validity is an ongoing process, other types of validity should be considered in order to gather more evidence of the quality and robustness of the CTC. While acknowledging the above limitations and the need to further consolidate the results, this study has shown some encouraging findings. Most importantly, it has demonstrated the feasibility to integrate different contexts, problem types, and item formats in assessing CT competency. Together, it is hoped that these findings can facilitate the process of theory development and assessment design in the emerging area of CT competency.

\section{CONCLUSION}

The emerging view of CT as a competency calls for a comprehensive assessment approach so as to capture its multifaceted nature. In this regard, competency-based assessments may be an 
appropriate assessment approach to measure CT competency in an integrative manner. It is a challenge, however, to include a wide range of problems and tasks in an assessment to reliably and validly represent the construct. This study has taken an initial step towards developing a robust assessment for this purpose. Overall, the items have shown appropriate and adequate coverage of different difficulty levels for a range of competency groups, especially those in the average to upper average performing groups. The results presented in this study have demonstrated some initial evidence of reliability and validity of the CTC albeit more evidence is needed using a larger sample size in future studies. Nonetheless, it is hoped that this study has contributed in two ways. First, this is one of the few studies that has investigated the feasibility to assess CT competency using an integrated approach. The findings shed light to the scope of the inferences that can be drawn from the contexts, item formats, and problem types introduced in the assessment. Lastly, it has taken a psychometric approach and rigorous analytical procedures to evaluate the quality and robustness of a CT assessment. The results can help facilitate assessment refinement and development.

\section{ACKNOWLEDGMENTS}

First and foremost, I am grateful to all the schools, teachers, and students who were involved in this study. My gratitude goes to Ceredig Cattanach-Chell, Ivo Timoteo, Stephen Trask (and Dahlia and Ethan Trask), as well as Tobias Kohn for the valuable feedback. Thanks must also be given to the anonymous reviewers who took their time in helping improve the article. I greatly appreciate Prof. Michelle Ellefson's continuous support in all my work.

\section{REFERENCE}

[1] V. Shute, C. Sun, and J. Asbell-Clarke. 2017. Demystifying computational thinking. Educational Research Review 22 https://doi.org/10.1016/j.edurev.2017.09.003

[2] A. L. S. O. Araujo, J. S. Santos, W. L. Andrade, D. D. S. Guerrero, and V. Dagienè. 2017. Exploring computational thinking assessment in introductory programming courses. 2017 IEEE Frontiers in Education Conference (FIE) 1-9. https://doi.org/10.1109/FIE.2017.8190652

[3] S. Grover and R. Pea. 2018. Computational thinking. A competency whose time has come. In Computer Science Education: Perspectives on Teaching and Learning, S. Sentence, E. Barendsen, and S. Carsten (Eds). Bloomsbury

[4] S. Papert. 1980. Mindstorms-Children, Computers and Powerful Ideas. New York: Basic Books, Inc.

[5] S. Papert and I. Harel. 1991. Constructionism. Norwood, NJ: Ablex Publishing.

[6] K. Brennan and M. Resnick. 2012. New frameworks for studying and assessing the development of computational thinking.

[7] M. G. Voskoglou and S. Buckley. 2012. Problem solving and computational thinking in a learning environment. ArXiv:1212.0750 [Cs]. http://arxiv.org/abs/1212.0750.

[8] S. Grover and R. Pea. 2013. Computational thinking in K-12: A review of the state of the field. Educational Researcher 42, 1 (2013), 38-43. https://doi.org/10.3102/0013189X12463051

[9] M. M. Sysło and A. B. Kwiatkowska. 2013. Informatics for all high school students. In Informatics in Schools. I. Diethelm \& R. T. Mittermeir (Eds.), Sustainable Informatics Education for Pupils of all Ages (43-56). Springer. https://doi.org/ 10.1007/978-3-642-36617-8_4

[10] A. Csizmadia, P. Curzon, M. Dorling, S. Humphreys, T. Ng, C. Selby, and J. Woollard. 2015. Computational thinking: A guide for teachers. (Available at: http://community.Computingatschool.org.uk/resources/2324). Unpublished report.

[11] J. Voogt, P. Fisser, J. Good, P. Mishra, and A. Yadav. 2015. Computational thinking in compulsory education: Towards an agenda for research and practice. Education and Information Technologies 20, 4 (2015), 715-728. https://doi.org/10. 1007/s10639-015- 9412-6

[12] J. M. Wing. 2006. Computational thinking. Communications of the ACM 49, 33-35. https://doi.org/10.1145/1118178. 1118215

[13] F. Kalelioglu, Y. Gulbahar, and V. Kukul. 2016. A framework for computational thinking based on a systematic research review. Baltic fournal of Modern Computing 4, 583-596.

[14] J. Cuny, L. Snyder, and J. Wing. 2010. Demystifying computational thinking for non-computer scientists. Unpublished Manuscript in Progress, Referenced in http://Www.Cs.Cmu.Edu/ CompThink/Resources/TheLinkWing.Pdf. 
[15] M. Webb, N. Davis, T. Bell, Y. J. Katz, N. Reynolds, D. P. Chambers, and M. M. Sysło. 2017. Computer science in K12 school curricula of the 2lst century: Why, what and when? Education and Information Technologies 22, 2 (2017), 445-468. https://doi.org/10.1007/s10639-016-9493-x

[16] P. J. Denning. 2009. The profession of IT. Beyond computational thinking. Communications of the ACM 52, 8 (2009), $28-30$

[17] M. Román-González, J.-C. Pérez-González, and C. Jiménez-Fernández. 2017. Which cognitive abilities underlie computational thinking? Criterion validity of the computational thinking test. Computers in Human Behavior 72, (2017) 678-691. https://doi.org/10.1016/j.chb.2016.08.047

[18] S. Bocconi, A. Chioccariello, G. Dettori, A. Ferrari, K. Engelhardt, P. Kampylis, and Y. Punie. 2016. Developing computational thinking in compulsory education. Implications for Policy and Practice. EUR - Scientific and Technical Research Reports. https://doi.org/10.2791/792158

[19] R. P. Y. Lai. 2021. Teachers' ontological perspectives of computational thinking and assessment: A text mining approach. Journal of Educational Computing Research, 07356331211043547. https://doi.org/10.1177/07356331211043547

[20] C. Selby and J. Woollard. 2013. Computational thinking: The developing definition [Monograph]. https://eprints. soton.ac.uk/356481/.

[21] V. Barr and C. Stephenson. 2011. Bringing computational thinking to K-12: What is involved and what is the role of the computer science education community? ACM Inroads 2, 1 (2011), 48-54. https://doi.org/10.1145/1929887.1929905

[22] J. Mueller, D. Beckett, E. Hennessey, and H. Shodiev. 2017. Assessing computational thinking across the curriculum. In Emerging Research, Practice, and Policy on Computational Thinking (251-267). P. J. Rich \& C. B. Hodges (Eds.) Cham, Switzerland: Springer International Publishing.

[23] Kazimoglu Cagin, Kiernan Mary, Bacon Liz, and MacKinnon Lachlan. 2012. Learning programming at the computational thinking level via digital game-play. Procedia Computer Science 9. 522-531. ISSN 1877-0509. https://doi.org/10. 1016/j.procs.2012.04.056

[24] National Research Council. 2010. Report of a Workshop on the Scope and Nature of Computational Thinking. Washington, DC: The National Academies Press. https://doi.org/10.17226/12840.

[25] X. Tang, Lin Q. Yin, R. Hadad, and X. Zhai. 2020. Assessing computational thinking: A systematic review of empirical studies. Computers \& Education 148103798.

[26] N. Alves, C. Wangenheim, and J. C. R. Hauck. 2019. Approaches to assess computational thinking competences based on code analysis in K-12 education: A systematic mapping study. Informatics in Education 18, 17-39. http://doi.org/ 10.15388/infedu.2019.02

[27] E. Wiebe, J. London, O. Aksit, B. W. Mott, K. E. Boyer, and J. C. Lester. 2019. Development of a lean computational thinking abilities assessment for middle grades students. In Proceedings of the 50th ACM Technical Symposium on Computer Science Education (456-461). ACM. https://doi.org/10.1145/3287324.3287390

[28] J. Moreno-León, G. Robles, and M. Román-González. 2016. Comparing computational thinking development assessment scores with software complexity metrics. 2016 IEEE Global Engineering Education Conference (EDUCON) 10401045. https://doi.org/10.1109/EDUCON.2016.7474681

[29] L. Werner, J. Denner, S. Campe, and D. C. Kawamoto. 2012. The fairy performance assessment: Measuring computational thinking in middle school. Proceedings of the 43rd ACM Technical Symposium on Computer Science Education 215-220. https://doi.org/10.1145/2157136.2157200

[30] K. H. Koh, A. Basawapatna, H. Nickerson, and A. Repenning. 2014. Real time assessment of computational thinking. 2014 IEEE Symposium on Visual Languages and Human-Centric Computing (VL/HCC) 49-52. https://doi.org/10.1109/ VLHCC.2014.6883021

[31] S. Grover. 2017. Assessing algorithmic and computational thinking in K-12: Lessons from a middle school classroom. In Emerging Research, Practice, and Policy on Computational Thinking (269-288). P. J. Rich \& C. B. Hodges (Eds.), Springer International Publishing. https://doi.org/10.1007/978-3-319-52691-1_17

[32] D. Weintrop and U. Wilensky. 2017. Comparing block-based and text-based programming in high school computer science classrooms. ACM Transactions on Computing Education (TOCE). https://dl.acm.org/doi/abs/10.1145/3089799

[33] M. Román-González, J.-C. Pérez-González, J. Moreno-León, and G. Robles. 2018. Can computational talent be detected? Predictive validity of the computational thinking test. International fournal of Child-Computer Interaction. https://doi.org/10.1016/j.ijcci.2018.06.004

[34] M. Román-González, J.-C. Pérez-González, and C. Jiménez-Fernández. 2017. Which cognitive abilities underlie computational thinking? Criterion validity of the computational thinking test. Computers in Human Behavior 72, 678-691. https://doi.org/10.1016/j.chb.2016.08.047

[35] J. Guggemos, S. Seufert, and M. Román-González Guggemos. 2020. Validity evidence for the "computational thinking test" at the upper-secondary level using IRT and CFA. American Education Researcher Association (AERE) Annual Meeting. San Francisco.

[36] S. C. Kong and Y. Q. Wang. 2021. Item response analysis of computational thinking practices: Test characteristics and students' learning abilities in visual programming contexts. Computers in Human Behaviours 122, 106835. 
[37] V. Dagiene and G. Stupuriene. 2016. Bebras-a sustainable community building model for the concept based learning of informatics and computational thinking. Informatics in Education 15, 1 (2016). https://eric.ed.gov/?id=EJ1097494.

[38] C. Izu, C. Mirolo, A. Settle, L. Mannila, and G. Stupuriene. 2017. Exploring Bebras tasks content and performance: A multinational study. Informatics in Education 16, 1 (2017), 39-59.

[39] M. Román-González, J. Moreno-León, and G. Robles. 2019. Combining assessment tools for a comprehensive evaluation of computational thinking interventions. In Computational Thinking Education S.-C. Kong \& H. Abelson (Eds.), (79-98). Springer. https://doi.org/10.1007/978-981-13-6528-7_6

[40] P. Hubwieser and A. Mühling. 2014. Competency mining in large data sets. Proceedings of the International foint Conference on Knowledge Discovery, Knowledge Engineering and Knowledge Management - Volume 1, 315-322. https: //doi.org/10.5220/0005129203150322

[41] T. Djambong and V. Freiman. 2016. Task-based assessment of students' computational thinking skills developed through visual programming or tangible coding environments. International Association for the Development of the Information Society. https://eric.ed.gov/?id=ED571389.

[42] O. Araujo, L. Wilkerson, D. Guerrero, and M. R. A. Melo. 2019. How many abilities can we measure in computational thinking?: A study on Bebras challenge. In Proceedings of the $50^{\text {th }}$ ACM Technical Symposium on Computer Science Education (SIGCSE'19). ACM, New York, NY, USA, 545-551.

[43] E. Rowe, Ma. V. Almeda, J. Asbell-Clarke, R. Scruggs, R. Baker, E. Bardar, and S. Gasca. 2021. Assessing implicit computational thinking in Zoombinis puzzle gameplay. Computers in Human Behavior 106707. https://doi.org/10. 1016/j.chb.2021.106707

[44] D. Basso, I. Fronza, A. Colombi, and C. Pahl. 2018. Improving assessment of computational thinking through a comprehensive framework. In Proceeding of the 18th Koli Calling International Conference on Computing Education Researcher $(1-5)$

[45] S. Grover. 2015. Systems of assessments for deeper learning of computational thinking in K-12. In Proceedings of the 2015 Annual Meeting of the American Education Research Association (AERA), 1-9

[46] S. Grover. 2020. Towards a framework for formative assessment of conceptual learning in K-12 computer science classrooms. In Proceedings of the 52nd ACM Technical Symposium on Computer Science Education (31-37).

[47] S.-C. Kong. 2019. Components and methods of evaluating computational thinking for fostering creative problemsolvers in senior primary school education. In Computational Thinking Education S.-C. Kong \& H. Abelson (Eds.), (119-141). Springer. https://doi.org/10.1007/978-981-13-6528-7_8

[48] W. Huang and C. L. Looi. 2020. A critical review of literature on "unplugged" pedagogies in K-12 computer science and computational thinking education. Computer Science Education 1-29.

[49] P. Hager, A. Gonczi, and J. Athanasou. 1994. General issues about assessment of competence. Assessment \& Evaluation in Higher Education 19, 1 (1994), 3-16. https://doi.org/10.1080/0260293940190101

[50] A. Gonczi. 1994. Competency based assessment in the professions in Australia. Assessment in Education: Principles, Policy \& Practice 1, 1 (1997), 27-44. https://doi.org/10.1080/0969594940010103

[51] CSTA \& ISTE. 2011. Operational Definition of Computational Thinking for K-12 Education. http://www.iste.org/docs/ pdfs/Operational-Definition-of-Computational-Thinking.pdf.

[52] C. Angeli, J. Voogt, A. Fluck, M. Webb, M. Cox, J. Malyn-Smith, and J. Zagami. 2016. A K-6 computational thinking curriculum framework: Implications for teacher knowledge. Journal of Educational Technology and Society 19, 3 (2016), 47-57. http://A K-6 Computational Thinking Curriculum Framework

[53] K. Koeppen, J. Hartig, E. Klieme, and D. Leutner. 2008. Current issues in competence modelling and assessment. Zeitschrift für Psychologie/fournal of Psychology 216, 60-72.

[54] A. Yadav, J. Good, J. Voogt, and P. Fisser. 2017. Computational thinking as an emerging competence domain. In Competence-based Vocational and Professional Education: Bridging the Worlds of Work and Education (1051-1067). Springer International Publishing. https://doi.org/10.1007/978-3-319-41713-4_49

[55] F. E. Weinert. 2001. Concept of competence: A conceptual clarification. In Defining and Selecting Key Competencies (45-65). Hogrefe \& Huber Publishers.

[56] S. Grover. 2021. Toward a framework for formative assessment of conceptual learning in K-12 computer science classrooms. In Proceedings of the 52nd ACM Technical Symposium on Computer Science Education

[57] D. Parsons and P. Haden. 2006. Parson's programming puzzles: A fun and effective learning tool for first programming courses. Proceedings of the 8th Australasian Conference on Computing Education - Volume 52, 157-163.

[58] B. J. Ericson, J. D. Foley, and J. Rick. 2018. Evaluating the efficiency and effectiveness of adaptive Parson's problems. Proceedings of the 2018 ACM Conference on International Computing Education Research - ICER'18, 60-68. https://doi. org/10.1145/3230977.3231000

[59] B. B. Morrison, L. E. Margulieux, B. Ericson, and M. Guzdial. 2016. Subgoals help students solve Parson's problems. SIGCSE 2016 - Proceedings of the 47th ACM Technical Symposium on Computing Science Education 42-47. https://doi. org/10.1145/2839509.2844617 
[60] B. J. Ericson, M. J. Guzdial, and B. B. Morrison. 2015. Analysis of interactive features designed to enhance learning in an ebook. Proceedings of the Eleventh Annual International Conference on International Computing Education Research 169-178. https://doi.org/10.1145/2787622.2787731

[61] J. M. Wing. 2017. Computational thinking's influence on research and education for all. Italian fournal of Educational Technology 25, 2 (2017), 7-14. DOI : 10.17471/2499-4324/922

[62] J. E. Pretz, A. J. Naples, and R. J. Sternberg. 2003. Recognizing, defining, and representing problems. In The Psychology of Problem Solving (3-30). J. E. Davidson \& R. J. Sternberg (Eds.), Cambridge University Press. https://doi.org/10.1017/ CBO9780511615771.002

[63] A. Labusch, B. Eickelmann, and M. Vennemann. 2019. Computational thinking processes and their congruence with problem-solving and information processing. In Computational Thinking Education (65-78). S.-C. Kong \& H. Abelson (Eds.), Springer. https://doi.org/10.1007/978-981-13-6528-7_5

[64] K. Aesaert, D. van Nijlen, R. Vanderlinde, and J. van Braak. 2014. Direct measures of digital information processing and communication skills in primary education: Using item response theory for the development and validation of an ICT competence scale. Computers \& Education 76, 168-181. https://doi.org/10.1016/j.compedu.2014.03.013

[65] I. Paek, K. Cole, and K. Cole. 2019. Using r for item response theory model applications. Routledge. https://doi.org/10. 4324/9781351008167

[66] W. J. Boone. 2016. Rasch analysis for instrument development: Why, when, and how? CBE Life Sciences Education 15, 4 (2016). https://doi.org/10.1187/cbe.16-04-0148

[67] D. Rizopoulos. 2006. ltm: An r package for latent variable modeling and item response analysis. Fournal of Statistical Software 17, 1 (2006), 1-25. https://doi.org/10.18637/jss.v017.i05

[68] A. Robitzsch, T. Kiefer, and M. Wu. 2018. TAM: Test analysis modules. R Package Version 2.9. https://CRAN.R- project. org/package=TAM.

[69] R. P. Chalmers. 2012. Mirt: A multidimensional item response theory package for the R environment. fournal of Statistical Software 48, 6 (2012), 1-29. DOI : 10.18637/jss.v048.i06

[70] D. Revicki. 2014. Internal consistency reliability. In Encyclopedia of Quality of Life and Well-Being Research. Michalos A.C. (eds) Springer, Dordrecht. http://doi.org/10.1007/978-94-007-0754-5_1494

[71] W. Revelle and R. E. Zinbarg. 2008. Coefficients alpha, beta, omega, and the glb: Comments on Sijtsma. Psychometrika 74, 1 (2008), 145. https://doi.org/10.1007/s11336-008-9102-z

[72] J. M. Linacre. 2002. What do infit and outfit, meansquare and standardized mean? Rasch Measurement Transactions 16, 2 (2002), 878-879.

[73] J. Kean, D. S. Brodke, J. Biber, and P. Gross. 2018. An introduction to item response theory and Rasch analysis of the eating assessment tool (EAT-10). Brain Impairment: A Multidisciplinary fournal of the Australian Society for the Study of Brain Impairment 19(Spec Iss 1), 91-102. https://doi.org/10.1017/BrImp.2017.31

[74] P. Kline. 2000. The Handbook of Psychological Testing (Second edition). London; New York : Routledge. https://trove. nla.gov.au/version/18879975.

[75] M. A. P. Souza, W. J. Coster, M. C. Mancini, F. C. M. S. Dutra, J. Kramer, and R. F. Sampaio. 2017. Rasch analysis of the participation scale (P-scale): Usefulness of the p-scale to a rehabilitation services network. BMC Public Health 17. https://doi.org/10.1186/s12889-017-4945-9

[76] A. B. Smith, R. Rush, L. J. Fallowfield, G. Velikova, and M. Sharpe. 2008. Rasch fit statistics and sample size considerations for polytomous data. BMC Medical Research Methodology 8, 1 (2008), 33. https://doi.org/10.1186/1471-2288-8-33

[77] H. Wickham. 2016. Ggplot2: Elegant Graphics for Data Analysis. Springer-Verlag New York. ISBN 978-3-319-24277-4. http://ggplot2.tidyverse.org.

[78] B. D. Wright and M. H. Stone. 1999. Measurement Essentials. Wilmington: Wide Range, Inc.

[79] S. Grover, R. Pea, and S. Cooper. 2016. Factors influencing computer science learning in middle school. In Proceedings of the 47th ACM Technical Symposium on Computing Science Education (552-557). ACM.

[80] D. Gürer and T. Camp. 2002. An ACM-W literature review on women in computing. ACM SIGCE Bulletin, Inroads, Special Issue: Women and Computing 34, 2 (2002), 121-127.

[81] P. Doerschuk, J. Liu, and J. Mann. 2007. Pilot summer camps in computing for middle school girls. ACM SIGCSE Bulletin 39, 3 (2007), 4. DOI : 10.1145/1269900.1268789

[82] J. Guggemos. 2021. On the predictors of computational thinking and its growth at the high-school level. Computers \& Education 161, Article 104060. http://doi.org/10.1016/j.compedu.2020.104060

[83] A. M. Hogenboom, F. J. Hermans, and L. J. Van der Maas. 2020. Computerized adaptive assessment of understanding of programming concepts in primary school children. Computer Science Education. http://doi.org/10.1080/08993408. 2021.1914461

Received March 2021; revised July 2021; accepted August 2021 